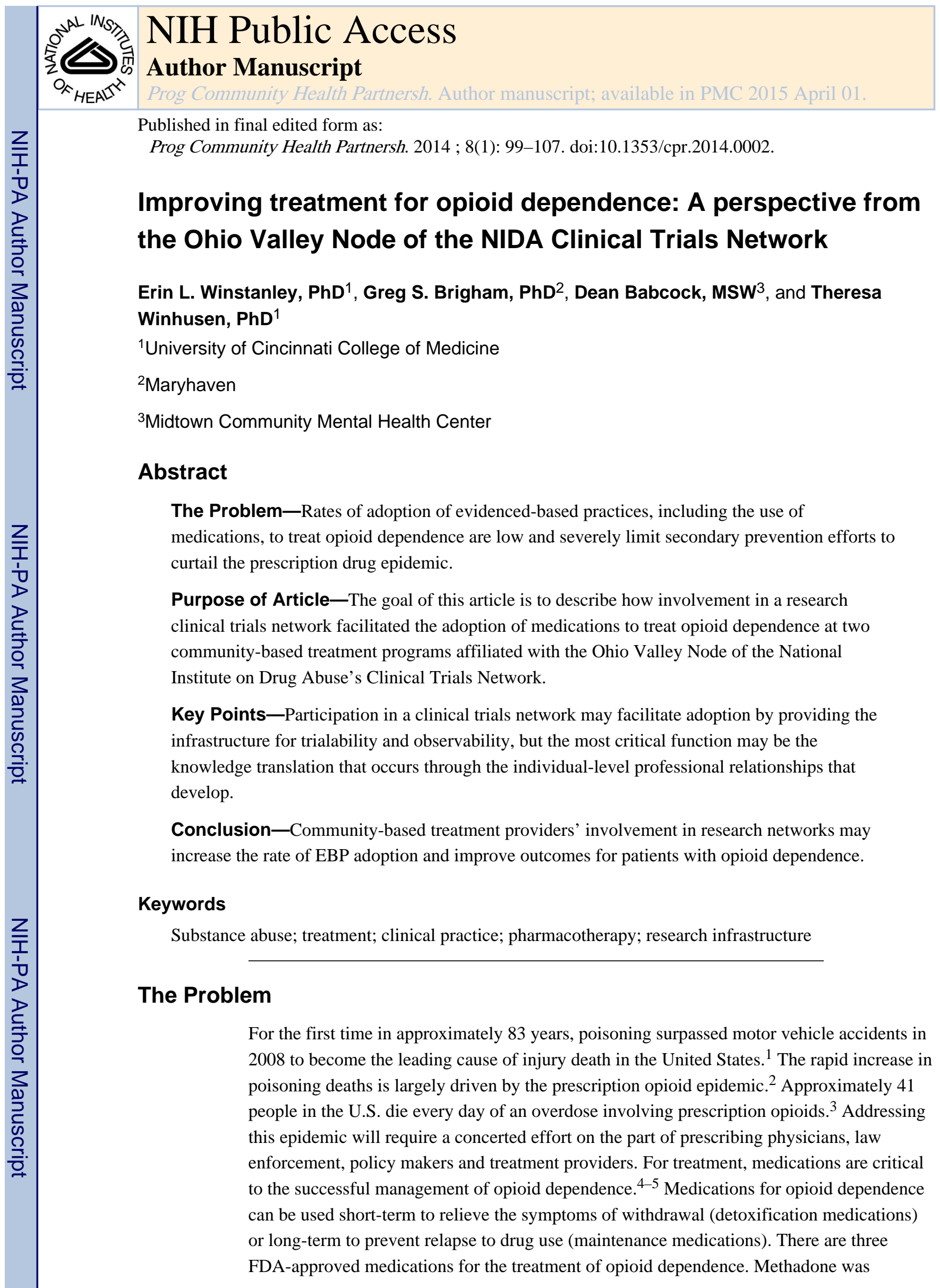


approved in 1972, the oral formulation of naltrexone was approved in 1994, buprenorphine was approved in 2002 and the depot formulation of naltrexone was approved in 2010. Patients treated with FDA-approved medications for opioid dependence are less likely to use illicit opioids, have a reduced risk of infectious diseases, are less likely to engage in criminal behaviors, have improved psychosocial functioning, and are less likely to die. ${ }^{6-12}$

In the U.S., approximately $11-34 \%$ of substance abuse treatment programs provide medications to treat opioid dependence. ${ }^{13-14}$ Among publically funded substance abuse treatment programs in the U.S., only $11.4 \%$ of programs provide methadone and $18.4 \%$ provide buprenorphine. ${ }^{13}$ There are several explanations for why there is limited use of medications to treat addictions: lack of financial resources to pay for addiction medications at the state, program, or patient levels; ${ }^{15-17}$ lack of medical staff with prescribing privileges $;{ }^{17}$ physicians' reluctance to prescribe medications for addictions; ${ }^{14,18-19}$ and regulatory dispensing barriers. ${ }^{5,17}$ For some treatment programs and clinicians, the barrier may be the philosophical belief that medications should not be used to treat addictions despite the overwhelming evidence of efficacy and effectiveness. ${ }^{20}$

It takes approximately 17 years for a clinical discovery to be integrated into routine medical practice ${ }^{21}$ hence there have been major efforts throughout medicine to increase the rate of adoption of evidence-based practices (EBPs). The National Institute on Drug Abuse (NIDA) formed the Clinical Trials Network (CTN) in 1999 to improve the quality of substance use disorder (SUD) treatment and to enhance adoption of EBPs for SUDs by establishing a unique collaboration between regional research and training centers (RRTCs), which are university based, and community-based treatment programs (CTPs). ${ }^{21}$ The NIDA CTN created the infrastructure for bi-directional collaboration between researchers and clinicians on research projects to ensure that the design, methods and findings would be highly relevant to community providers. To date, the NIDA CTN has conducted over 50 clinical trials and has enrolled over 16,000 study participants.

The NIDA CTN is very similar to other national practice-based research networks (PBRN), however it does not meet the Agency for Healthcare Research and Quality's (AHRQ) definition of a primary care PBRN because less than 50 percent of the membership is comprised of primary care clinicians. The NIDA CTN focuses on the improvement of treatment for SUD and community provider participation is not restricted by degree specialization, degree level or setting of clinical services. The NIDA CTN is further differentiated from most PBRNs given that the primary focus is on conducting clinical trials, although the network does serve as platform for other types of research.

Currently the NIDA CTN has 13 nodes representing RRTCs that facilitate and coordinate research projects with $183 \mathrm{CTPs}$ where the research projects may be conducted. The NIDA CTN has CTPs in 42 states and CTPs include providers across a variety of treatment settings such as the specialty addiction treatment system, primary care, HIV/infectious disease clinics and emergency departments. The NIDA CTN is also compromised of a clinical coordinating center at NIDA and a data/statistical center that manages and analyzes the study data. The NIDA CTN has an annual meeting that brings together RRTC and CTP representatives across the country to collaborate on the development of new and existing 
research projects. The goal is to equally involve the university and community partners in the research process, which is reflected in the organizational infrastructure lead by a Steering Committee that is co-chaired by one elected RRTC and CTP representative. The CTP's have their own committee that meets in-person at the annual meeting and maintains communication throughout the year via a listserv and monthly conference calls. CTPs also have the opportunity to develop manuscripts, present study findings and develop dissemination materials. CTPs receive financial reimbursement for participation in the national and node-level conference calls, as well as research-related funds to support participation in specific CTN studies. Additionally, CTPs that participate in CTN studies have the opportunity for their staff to receive free clinical and research training.

The recruitment of CTPs into the CTN varies at the node-level. The OVN, in its initial application to join the $\mathrm{CTN}$, identified addiction treatment programs in the surrounding geographic area with a medium to large patient population that represented different levelsof-care including residential, intensive outpatient treatment and standard outpatient treatment. The Principal Investigators would contact the addiction treatment program and if the agency was interested in collaborating on research studies, an in-person meeting would be scheduled. Additional CTPs may be recruited into the CTN based on specific research projects and the participant inclusion criteria associated with that study (e.g., number of new patients that weekly present seeking treatment for cocaine dependence). As the CTN has developed over the years and addiction treatment services are increasingly available in the non-addiction specialty treatment system, the CTN has begun to recruit CTPs in general medical settings such as primary care and emergency departments. The Ohio Valley Node (OVN) is one of the RRTCs of the NIDA CTN and this node includes 23 CTPs in 15 states in the Midwest region of the U.S.

\section{Purpose of Article}

The goal of this article is to describe how collaboration between clinicians and researchers in a clinical trials network facilitated the adoption of medications to treat opioid dependence at two community-based treatment programs affiliated with the OVN of the NIDA CTN. Both of the treatment programs are large not for profit facilities that treat patients with either public or private insurance. One program (Maryhaven in Columbus, Ohio) had limited experience using FDA-approved medications to treat opioid dependence prior to joining the NIDA CTN, while the second (Midtown in Indianapolis, Indiana) had a methadone clinic but no experience with buprenorphine or naltrexone. This article will discuss the adoption of medications to treat opioid dependence by highlighting critical turning points, lessons learned and challenges that were encountered. The discussion will be framed by Rogers's theory of diffusion of innovation and within the context of Damschroder and Hagedorn's (2011) consolidated framework for implementation research. This article is co-authored by two members of the OVN RRTC and two members of OVN CTPs. The OVN CTP representatives wrote the section on their respective treatment agencies and the node representatives wrote the other sections. All of the authors edited and reviewed the manuscript prior to submission. 


\section{Key Points}

\section{Adoption of Medications at Maryhaven}

Maryhaven is central Ohio's oldest and largest addiction treatment facility with admissions of over 7,000 annually. Prior to joining the NIDA CTN, there were no specific empirically supported addiction treatment practices being used at Maryhaven. Maryhaven primarily used a 12-Step based approach to psychosocial treatment. ${ }^{23}$ Clinical experience, rather than science, most frequently informed decisions about treatment options or how to address organizational challenges. Clonidine was being used in a 7-day residential detoxification program to ease the symptoms of opioid withdrawal, however maintenance medications were not being used to prevent relapse to drug use. Approximately $50 \%$ of patients completed the residential detoxification program and only $30 \%$ of those transitioned from detoxification to outpatient treatment. Among the 30\% that initiated outpatient treatment, few completed the outpatient treatment program and nearly all of the patients relapsed to opioid use. At that time, there was considerable skepticism of methadone throughout the organization including the governing board, the administration and staff at Maryhaven, as it was seen as simply switching the patient to a new and potentially more severe drug dependence.

Upon joining the OVN in 1999, Maryhaven was offered the opportunity to participate in one of three research projects and the medical director at that time wanted to participate in the buprenorphine taper protocol because she felt that Maryhaven was not adequately treating patients with opiate dependence. Maryhaven participated in the residential detoxification study (NIDA CTN-0001) comparing buprenorphine versus clonidine (the standard practice at the time) and was one of 12 CTPs nationally that participated in the study. Maryhaven staff were trained to collect standardized clinical assessments including the objective assessment of opiate withdrawal, the collection of urine drug screens and state-of-the-art techniques to manage opiate withdrawal with medications. Additionally, Maryhaven staff conducted research activities such as study consent, data collection, and data entry. Throughout the course of the study, Maryhaven received technical assistance, both on site and through teleconferences, from the OVN and participated in weekly conference calls with the national research team. The results of the study were immediately clear. A counselor working in the detoxification program at the time commented, "It [buprenorphine] is remarkable. You can tell the difference just by walking down the hall, the clonidine patients are throwing-up and the buprenorphine patients are ready for treatment." Staff involved with the CTN-0001 study were persuaded that medications should be adopted and they became local champions working with the Maryhaven Executive Staff and the Board of Trustees to expand adoption of medications. Additionally, the staff used the results from the NIDA CTN-0001 study to receive initial funding from the Columbus Medical Association Foundation and the Alcohol, Drug and Mental Health Board of Franklin County to implement the buprenorphine taper studied in CTN-0001 at Maryhaven. Maryhaven adopted the use of buprenorphine as a medication for detoxification from opioids in 2003 soon after buprenorphine received FDA approval. ${ }^{23}$ 
Adoption of EBPs requires more than just knowledge of the treatment; it often requires additional training and the EBP may need to be tailored at the program level. In the process of participating in the CTN-0001 study, Maryhaven staff were trained how to induct, dose and taper patients on buprenorphine. Additionally, clinical staff were trained to conduct standardized clinical assessments that measured opioid withdrawal, which is important to ensure that the dose relieves withdrawal symptoms without causing over-sedation.

Maryhaven used this training and first-hand clinical experience using buprenorphine to tailor the CTN-0001 taper schedule for their patient population. ${ }^{23}$ Upon implementation of buprenorphine as medication for detoxification, the leadership at Maryhaven was able to realize that they had the organizational capacity to implement medication assisted treatments and that medications could improve outcomes for their patients. However, Maryhaven's use of buprenorphine as a detoxification medication was not sufficient to quell ambivalence among staff regarding the use of medications to prevent relapse and one counselor said, "I know it works but I worry that it is too easy." Still, the success with buprenorphine as a medication for detoxification was sufficient to motivate the administration and staff to continue a dialogue on the utility of longer durations of maintenance medications to prevent relapse.

A major milestone was when Maryhaven adopted methadone and opened an outpatient opiate treatment program. This happened through a lengthy contentious process that was sustained by the trusting relationships that developed in the NIDA CTN between investigators and treatment professionals working side-by-side to improve treatment. One example of this occurred following the events of September 11, 2001. At this time, NIDA CTN members were in Washington DC for a national meeting and the resulting airport closures resulted in the entire OVN traveling back home on a charter bus. This long bus ride provided ample opportunity for a Maryhaven clinician to discuss the clinical experience of using addiction medications, such as methadone with a trusted research colleague. This pivotal moment committed this clinician at Maryhaven, to implementing medications for both detoxification and relapse prevention.

In the 13 years since Maryhaven joined the NIDA CTN, Maryhaven has inducted nearly 10,000 patients onto medications for opioid dependence and now uses all of the FDAapproved medications to treat opioid dependence. It took approximately two years after beginning CTN-0001 for Maryhaven to initially implement buprenorphine and an additional two years for broader adoption of MAT. Maryhaven leadership was compelled to support MAT because of the obvious clinical improvements in Maryhaven patients and that adoption would not require additional costly clinical training, as this was provided at no cost as part of participation in the CTN-0001 study. The personal experiences of clinical staff and patients eventually overcame any philosophical barriers to medication adoption.

Maryhaven's professional staff have become advocates for statewide efforts to improve access to medications to treat opioid dependence. The opiate treatment program director at Maryhaven, who is also a primary care physician at another location, has described working in the opiate treatment program as the most rewarding area of his work because he routinely has patients, in tears, thanking him for helping to save their lives. While Maryhaven has made significant strides to adopt science-based practices, the financial barriers to expanding access to addiction medications throughout all of their treatment locations has been 
challenging. Ohio does not have a mechanism to provide expensive medications at a reduced cost or for free for patients without a payor source and therefore provision of MAT to this patient population may not be feasible unless the patient is able to pay out-of-pocket. However, it should be noted that the State is currently working towards expanding access to MAT and Maryhaven clinical staff, as well as OVN staff, have served as clinical and scientific experts during this process.

\section{Adoption of Medications at Midtown}

Midtown had been a leader in addiction treatment in Indiana by operating the first methadone treatment program in the State, and having a history of offering ambulatory detoxification for opioid dependence for many years. Midtown Community Mental Health Center's Addiction Services initially wanted to join the OVN of the NIDA CTN so that they could become partners in the conduct of clinical research studies and because they wanted to improve outcomes for their patients. Historically, Midtown had participated in numerous research and evaluation projects, but such efforts were limited to participant recruitment for researchers outside of their treatment system and Midtown staff were not involved in these research projects. The NIDA CTN allowed Midtown to be an active participant in the design, implementation and collection of research data. Midtown's first experience using buprenorphine was in the NIDA CTN study (CTN-0002) that compared buprenorphine versus clonidine for outpatient detoxification. Almost immediately after joining the CTN, Midtown had the opportunity to begin work on the CTN-0002 study which was an outpatient detoxification study comparing buprenorphine to treatment as usual (TAU). Midtown staff were identified to work on the study and were trained on the research and clinical protocols. This would be Midtown Addiction Services' first attempt at operating a scientifically based research protocol. Midtown staff learned how to recruit, consent and administer research documents to study participants. Staff successfully implemented the protocol with assistance from the OVN Node and the national team leading the study. Midtown patients were very positive towards the opportunity to participate in the study and the study attracted patients from outside Midtown's standard service area. Soon after study enrollment began, it became very clear as to which subjects were randomized to receiving buprenorphine versus TAU. Patients responded quickly to buprenorphine and it was clinically obvious that buprenorphine was superior at relieving opiate withdrawal symptoms. Staff were able to easily recognize the positive attributes of using buprenorhine for the detoxificaion of opiate dependent patients.

Midtown's experience using this new medication during the CTN study was very successful and buprenorphine was perceived as a positive treatment option. Treatment programs with no experience using psychiatric or addiction medications are less likely to adopt new medications for SUDs. ${ }^{13}$ While Midtown had adopted methadone prior to joining the NIDA CTN, the adoption of buprenorphine was complicated and took longer than anyone anticipated, in part, because Midtown is part of a large medical system that includes a public hospital. 


\section{Challenges at Midtown}

Administrative and clinical leadership at Midtown were convinced that new treatment strategies were needed to improve outcomes for patients with SUDs. Midtown leadership were very supportive of expanding the use of addiction medications, however other structural barriers presented significant challenges. The first challenge that Midtown faced was they did not have a physician, outside of the methadone clinic, with specialty training in addiction medicine who could champion and spearhead the use of new medications. It took several years for Midtown to recruit additional addiction-trained physicians.

The second challenge was that a large proportion of patients at Midtown are uninsured or publically insured, therefore Midtown needed to identify a mechanism to pay for buprenorphine. Midtown was not able to use the patient payment system used in the methadone program because buprenorphine costs significantly more than methadone. When Midtown initiated the 13-day buprenorphine detoxification program within the methadone clinic, they needed to set-up a fee structure that took time to develop. The use of buprenorphine outside of the methadone clinic presented other patient payment system problems and required getting buprenorphine approved on the larger hospital formulary system. Public hospitals with limited resources need to conduct a cost benefit analysis and develop practice parameters that define how the medication can be used within its system prior to adding new medications to their formularies. Close scrutiny must be paid to costly medications and the cost difference between methadone and buprenorphine is significant.

The third challenge was that because Midtown is part of a large medical system, adoption would require buy-in across this medical system. The medical system's leadership needed to conduct an analysis in order to consider alternative implementation strategies for adoption and the development of guidelines that would ensure appropriate use of these medications across the medical system.

In 2010, buprenorphine and depot naltrexone were added to the general formulary for use by physicians with specialty training in addiction medicine and this occurred approximately nine years after Midtown participated in CTN-0002. A critical factor in getting these medications added to the formulary was that Midtown participated in the CTN-0002 study and knew first-hand that this medication improved outcomes for their patients. Pharmacists, within their treatment system who had specialty training in psychiatry, became local champions for adoption of these medications and worked to provide compelling data and research that was pivotal in this process and helped to obtain the approval needed.

Midtown's efforts to expand use of addiction medications were supported by the Indiana Division of Mental Health and Addiction, who sponsored workshops in the region and referenced Midtown as "experts" to serve as an example for adopting medication assisted treatment to other treatment programs in the State. However, the delay in buprenorphine adoption at Midtown was not clearly understood by other regional treatment programs. Some assumed that Midtown was not adopting buprenorphine because the medication was not effective, and therefore were not considering adoption of buprenorphine within their own treatment programs. Ultimately, the leadership at Midtown had to address this misperception and publically explain the reason for the delay in adoption. 
Regulatory issues and the development of a billing process further prolonged buprenorphine adoption at Midtown. Opioid Treatment Programs (OTPs) are regulated at the federal and state-levels. Midtown worked with the Single State Authority in Indiana to determine how to run a buprenorphine clinic within the state's existing OTP regulations, which were originally developed for dispensing methadone. Many of the regulations for dispensing methadone can present a burden to patients and create barriers to accessing methadone. Therefore Midtown worked hard, in collaboration with state officials, to ensure that the use of buprenorphine within the OTP regulations were not overly restrictive.

Midtown adopted the outpatient detoxification protocol used during the CTN-0002 study in 2009 within their OTP clinic. Soon after adopting buprenorphine as an OTP detoxification medication, it became clear that Midtown's patients wanted long-term access to the medication to prevent relapse to opioid use. The OTP program, providing the detoxification medications, had easy access to the medication through its onsite pharmacy. However, providing buprenorphine as a maintenance medication for patients in non- OTP outpatient addiction programs presented another set of challenges. Midtown's outpatient addiction treatment programs do not have on-site pharmacy services and mechanisms for medication storage, medication dispensing, dose monitoring, diversion prevention, nor appropriate medical personnel. Midtown has diligently worked through these implementation challenges and now methadone, buprenorphine, and depot naltrexone are used in addition to psychosocial therapeutic interventions.

\section{Conclusions}

Research suggests that participation in the NIDA CTN facilitates adoption of EBPs for SUDs. ${ }^{24}$ Research has found that SUD treatment programs within the NIDA CTN are two to three times more likely to adopt buprenorphine. ${ }^{25-26}$ CTPs that participated in the NIDA CTN buprenorphine clinical trials are even more likely to have adopted buprenorphine. ${ }^{14,25}$ Maryhaven and Midtown are two community-based treatment programs that were motivated to adopt medications for the treatment of opioid dependence by their involvement in a clinical trials network and were able to sustain efforts to overcome many of the known barriers to medication adoption.

Based on Rogers's theory on the diffusion of innovations, five factors that facilitate adoption of innovations are relative advantage, trialability, observability, compatibility, and simplicity. ${ }^{27}$ Leadership and staff at both CTPs believed that outcomes for opioid treatment could be improved (perceived need for benefit) and participated in clinical studies, which provided the opportunity for trialability and observability. Both CTPs were able to gain the clinical skills necessary for adoption and to obtain data that demonstrated improvement in patient outcomes at their respective treatment programs by participating in the NIDA CTN (observability). Compatibility of opioid medications can present a barrier at the philosophical and structural levels. Lack of buy-in from clinic staff and leadership is frequently reported as a significant barrier to medication adoption. ${ }^{14,19-20}$ An abstinencebased recovery approach to addiction treatment was a major barrier at Maryhaven that was, ultimately, addressed by an incremental process of adoption that resulted in a medication assisted recovery philosophy. However, philosophical barriers to medication use were not a 
barrier at Midtown may be due to Midtown being part of a larger medical system where medications are used on a routine basis to treat psychiatric and medical conditions. Adoption of medications within the addiction specialty treatment system is not simplistic, given that outpatient treatment programs infrequently have the medical staff needed to prescribe medications. ${ }^{17}$ The ability to adopt evidence-based medications to treat addiction requires knowledge of induction, dosing, tapering; clinicians that can and will prescribe; methods to pay for medications; and technical assistance for clinically complicated patients.

Table 1 provides a summary of implementation factors, barriers to MAT adoption, and pivotal factors in MAT adoption. The implementation factors are taken from Damschroder and Hagedorn's (2011) consolidated framework for implementation research and includes the core elements of Rogers's theory of diffusion of innovations. At both sites, the intervention demonstrated positive outcomes that were observable by patients, clinicians and administrators. Participation in the NIDA CTN provided clinical training and taught community-based treatment providers procedures to administer MAT which significantly reduces the cost of adoption. The NIDA CTN and other PBRNs provide opportunities for community providers to 1) gain first-hand experience with new interventions, 2) observe whether these interventions improve outcomes for their own patients and 3) remove barriers related to clinical training or procedural knowledge that may be associated with the intervention. Furthermore, research networks may provide a professional context within which adoption of EBPs is the cultural norm and provide informal opportunities for community-based providers to learn about complex interventions. Additional research is needed to evaluate whether the CTN and other PBRNs foster the development of trusting professional relationship that facilitate adoption.

The NIDA CTN is a research network that facilitates the adoption of innovative sciencebased treatments for SUDs. One of the intrinsic values of participating in a research network is that the infrastructure allows opportunities for collaborative relationships to develop, building trust over time, and ultimately providing a professional network that can provide technical assistance that may be the final barrier to adoption or service as a tipping point for adoption. The importance of face-to-face social relationships in the adoption of innovative strategies has been demonstrated by the Agricultural Extension Services that was built on the understanding that innovation occurs in local communities amongst people that are familiar and trusted. ${ }^{28}$ Participation in a clinical trials network may facilitate adoption of innovative health care practices by providing the infrastructure for trialability and observability, but the most critical aspect of these types of research and practice-based partnerships may be the knowledge translation that occurs through the individual-level professional relationships that develop.

\section{REFERENCES}

1. Paulozzi L, Dellinger A. Degutis. Lessons from the Past. Inj Prev. 2012; 18:70. [PubMed: 22210643]

2. Paulozzi LJ, Weisler RH, Patkar AA. A national epidemic of unintentional prescription opioid overdose deaths: how physicians can help control it. J Clin Psychiatry. 2011; 72:589-592. [PubMed: 21536000] 
3. Centers for Disease Control and Prevention. Prescription Painkiller Overdoses in the US. 2011. (Vital signs November 2011). Retrieved from http://www.cdc.gov/vitalsigns/PainkillerOverdoses/ index.html.

4. Veilleux JC, Colvin PJ, Anderson J, York C, Heinz AJ. A review of opioid dependence treatment: Pharmacological and psychosocial interventions to treat opioid addiction. Clin Psychol Rev. 2010; 30:155-166. [PubMed: 19926374]

5. Kresina TF, Lubran R. Improving public health through access to and utilization of medication assisted treatment. Int J Environ Res Public Health. 2011; 8:4102-4117. [PubMed: 22073031]

6. Clark RE, Samnaliev M, Baxter JD, Leung GY. The evidence doesn't justify steps by state Medicaid programs to restrict opioid addiction treatment with buprenorphine. Health Affairs. 2011; 30(8):1425-1431. [PubMed: 21821560]

7. Ward J, Hall W, Mattick RP. Role of maintenance treatment in opioid dependence. Lancet. 1999; 353(9148):221-226. [PubMed: 9923893]

8. McLellan AT, Arndt IO, Metzger DS, Woody GE, O'Brien CP. The effects of psychosocial services in substance abuse treatment. JAMA. 1993; 269(15):1953-1959. [PubMed: 8385230]

9. Johansson BA, Berglund M, Lindgren A. Efficacy of maintenance treatment with naltrexone for opioid dependence: a meta-analytical review. Addiction. 2006; 101(4):491-503. [PubMed: 16548929]

10. Martin WR, Jasinski DR, Mansky PA. Naltrexone, an antagonist for the treatment of heroin dependence. Effects in man. Arch. Gen. Psychiatry. 1973; 28(6):784-791. [PubMed: 4707988]

11. Comer SD, Sullivan MA, Yu E, Rothenberg JL, Kleber HD, Kampman K, Dackis C, O'Brien CP. Injectable, sustained-release naltrexone for the treatment of opioid dependence: a randomized, placebo-controlled trial. Arch. Gen. Psychiatry. 2006; 63(2):210-218. [PubMed: 16461865]

12. Weiss RD, Potter JS, Fiellin DA, Byrne M, Connery HS, Dickinson W, Gardin J, Griffin ML, Gourevitch MN, Haller DL, Hasson AL, Huang Z, Jacobs P, Kosinski AS, Lindblad R, McCanceKatz EF, Provost SE, Selzer J, Somoza EC, Sonne SC, Ling W. Adjunctive counseling during brief and extended buprenorphine-naloxone treatment for prescription opioid dependence. Arch. Gen. Psychiatry. 2011; 68(12):1238-1246. [PubMed: 22065255]

13. U.S. Department of Health and Human Services, Substance Abuse and Mental Health Services, Administration Office of Applied Studies. 2010 State profile — United States national survey of substance abuse treatment services (N-SSATS). 2011. Retrieved from http:// wwwdasis.samhsa.gov/webt/state_data/US10.pdf.

14. Knudsen HK, Abraham AJ, Roman PM. Adoption and implementation of medications in addiction treatment programs. J Addict Med. 2011; 5:21-27. [PubMed: 21359109]

15. Horgan CM, Reif S, Hodgkin D, Garnick DW, Merrick EL. Availability of addiction medications in private health plans. J Subst Abuse Treat. 2008; 34:147-156. [PubMed: 17499959]

16. Ducharme LD, Abraham AJ. State policy influence on the early diffusion of buprenorphine in community treatment programs. Subst Abuse Treat Prev Policy. 2008; 3:17. [PubMed: 18570665]

17. Martino S, Brigham GS, Higgins C, Gallon S, Freese TE, Albright LM, Hulsey EG, Krom L, Storti SA, Perl H, Nugent CD, Pintello D, Condon TP. Partnerships and pathways of dissemination: The National Institute on Drug Abuse - Substance Abuse and Mental Health Services Administration Blending Initiative in the Clinical Trials Network. J Subst Abuse Treat. 2010; 38:S31-S43. [PubMed: 20307793]

18. Albright J, Ciaverelli R, Essex A, Tkacz J, Ruetsch C. Psychiatrist characteristics that influence use of a buprenorphine medication-assisted treatment. J Addict Med. 2010; 4(4):197-203. [PubMed: 21769038]

19. West JC, Kosten TR, Wilk J, Svikis D, Triffleman E, Rae DS, Narrow WE, Duffy FF, Regier DA. Challenges in increasing access to buprenorphine treatment for opiate addiction. Am J Addict. 2004; 13:S8-S16. [PubMed: 15204672]

20. Riekmann T, Kovas AE, Rutkowski BA. Adoption of medications in substance abuse treatment: Priorities and strategies of single state authorities. J Psychoactive Drugs. 2010 Sep.(Supp 6):227238. [PubMed: 21138199]

21. Balas EA. From appropriate care to evidence-based medicine. Pediatr Ann. 1998; 27(9):581-584. [PubMed: 9778710] 
22. Tai B, Straus MM, Liu D, Sparenborg S, Jackson R, McCarty D. The first decade of the National Drug Abuse Treatment Clinical Trials Network: Bridging the gap between research and practice to improve drug abuse treatment. J Subst Abuse Treat. 2010; 38(Suppl 1):S4-S13. [PubMed: 20307794]

23. Brigham GS, Amass L, Winhusen T, Harrer JM, Pelt A. Using buprenorphine short-term taper to facilitate early treatment engagement. J Subst Abuse Treat. 2007; 32:349-356. [PubMed: 17481458]

24. Roman PM, Abraham AJ, Rothrauff TC, Knudsen HK. A longitudinal study of organizational formation, innovation adoption and dissemination activities within the National Drug Abuse Treatment Clinical Trials Network. J Subst Abuse Treat. 2010; 38:S44-S52. [PubMed: 20307795]

25. Durcharme LJ, Knudsen HK, Roman PM, Johnson JA. Innovation adoption in substance abuse treatment: Exposure, triability, and the Clinical Trials Network. J Subst Abuse Treat. 2007; 32:321-329. [PubMed: 17481455]

26. Ducharme LJ, Roman PM. Opioid treatment programs in the Clinical Trials Network: Representativeness and buprenorphine adoption. J Subst Abuse Treat. 2009; 37:90-94. [PubMed: 19004597]

27. Rogers, EM. Diffusion of innovations. 4th ed.. New York: The Free Press; 1995.

28. Berwick DM. Disseminating innovations in health care. JAMA. 2003; 289(15):1969-1975. [PubMed: 12697800]

29. Damschroder LJ, Hagedorn HJ. A guiding framework and approach for implementation research in substance use disorders treatment. Psychology of Addictive Behaviors. 2011; 25(2):194-205.

[PubMed: 21443291] 


\section{Table 1}

Framework of MAT Adoption with the NIDA CTN

\begin{aligned} & \hline \multicolumn{2}{c}{ Implementation Factors $^{a}$} \\ & \hline I. Intervention characteristics \\ & a. $\quad$ Intervention source \\ & b. $\quad$ Evidence \& quality \\ & c. $\quad$ Relative advantage \\ & d. $\quad$ Adaptability \\ & e. Trialability \\ & f. Complexity \\ & g. Design quality \& \\ & h. packaging \end{aligned}

Barriers to MAT Adoption

- Many outpatient treatment programs have no experience using medications

- Adoption of medication may require significant costs to train staff in clinical procedures \& costs to deliver the medications

- $\quad$ Treatment programs without experience using medications may not have a mechanism to bill for medication-related expenses
OVN CTPs

Trialability achieved through study participation in the NIDA CTN

- $\quad$ NIDA CTN provided the opportunity to generate local evidence of intervention quality \& outcomes

- Relative advantage of medication versus standard approaches was compelling

- $\quad$ Cost \& complexity addressed during study

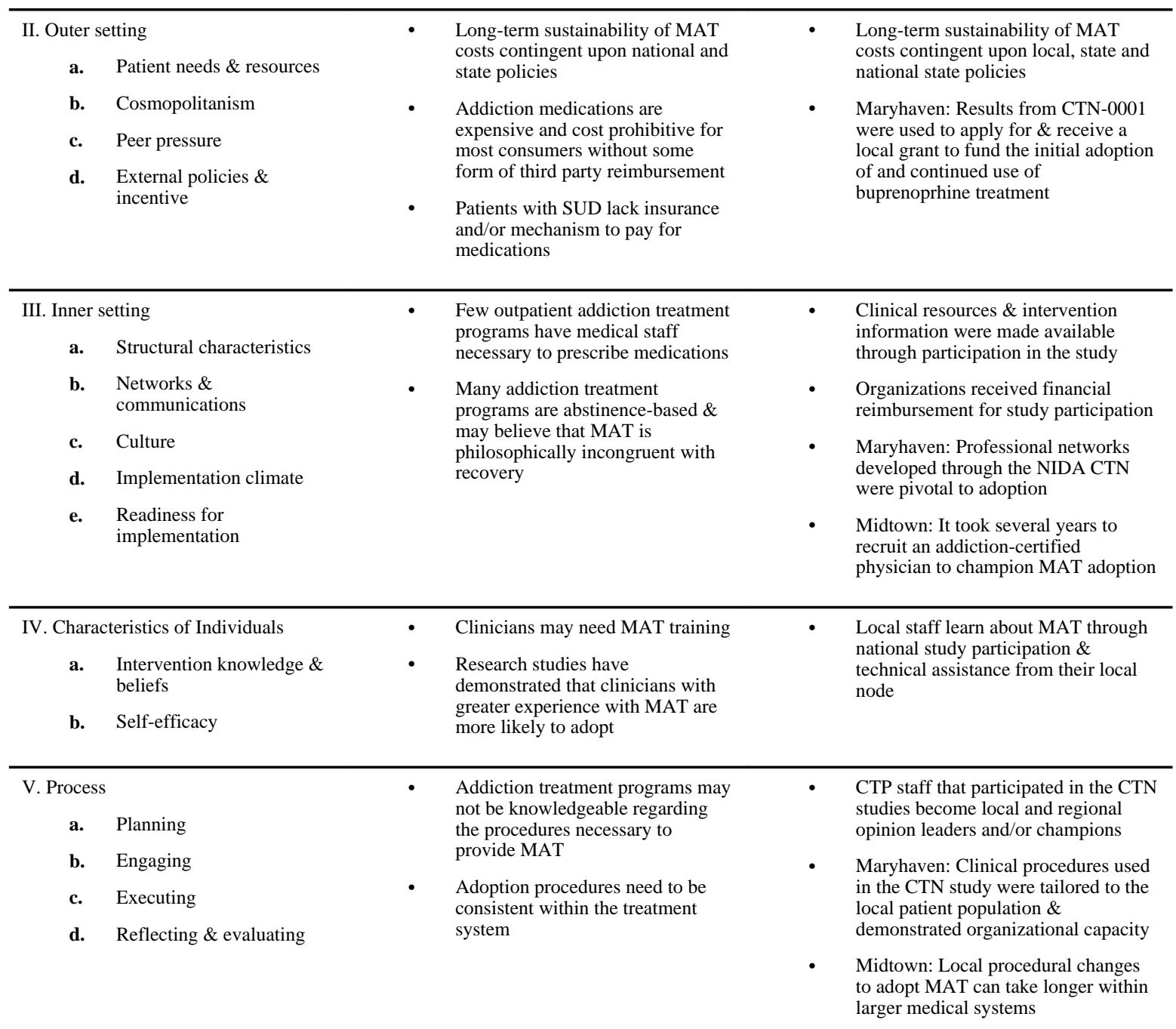

Prog Community Health Partnersh. Author manuscript; available in PMC 2015 April 01. 
`Factors influencing implementation of MAT based on Damschroder \& Hagendon's (2011) Consolidated Framework for Implementation Research. $^{29}$ 